\title{
LETTER
}

\section{Whole blood impedance aggregometry findings in experimental endotoxinemia}

\author{
Vivian Simm, Daniel Dirkmann, Jürgen Peters and Matthias Hartmann* \\ See related research by Adamzik et al., http://ccforum.com/content/16/5/R204
}

In an observational study in a recent issue of Critical Care, we demonstrated that platelet function, as assessed by impedance aggregometry, is reduced in patients with severe sepsis and poor prognosis [1]. In the present study, we investigated whether the prototypic endotoxin lipopolysaccharide (LPS) (Escherichia coli; serotype 0.111:B4; Sigma-Aldrich, Munich, Germany) exerts comparable effects in an in vitro model. Therefore, heparinized blood samples were drawn from the antecubital vein of healthy probands. After incubation of samples with LPSs $(100 \mu \mathrm{g} /$ $\mathrm{mL}$ ) or vehicle for 240 minutes at $37^{\circ} \mathrm{C}$, samples were subjected to impedance aggregometry (Roche Diagnostics Deutschland GmbH, Mannheim, Germany), and aggregation was induced by adenosine diphosphate (ADP), collagen, thrombin receptor-activating peptide (TRAP), or arachidonic acid.

Incubation of whole blood samples with LPS markedly inhibited platelet function as revealed by determination of the impedance aggregometry variable of area under curve (expressed in arbitrary units). In LPS-treated samples, platelet function was significantly reduced in comparison with vehicle-treated samples when collagen $(-55 \%, P=0.008)$, ADP $(-45 \%, P=0.008)$, or TRAP $(-20 \%, P=0.04)$ served as the activator. In contrast, no LPS-induced decrease in platelet function was detectable when arachidonic acid was used as the activator (Figure 1).

Our in vitro findings on LPS-induced inhibition of platelet aggregation closely agree with our recent observational study in patients with severe sepsis. In both studies, the decrease in platelet function was dependent on the activator used and the ranking of activators was identical (collagen > TRAP > ADP). Moreover, our finding that LPS did not reduce arachidonic acid-induced platelet aggregation is in agreement with our previous observational study. In contrast to the other activators, arachidonic acid was neither an independent predictor of the diagnosis of severe sepsis nor an independent predictor of the outcome in severe sepsis.

Thus, the present study demonstrates that the decrease in platelet aggregation seen in patients with severe sepsis can be mimicked in the in vitro setting, suggesting comparable pathophysiological mechanisms.

\section{Abbreviations \\ ADP, adenosine diphosphate; LPS, lipopolysaccharide; TRAP, thrombin receptor-activating peptide.}

\section{Competing interests}

The authors declare that they have no competing interests.

\section{Acknowledgments}

Approval of the ethics committee (University Hospital Essen) and consent of the healthy probands were obtained. The authors declare that funding for this report was solely departmental.

\section{Published: 17 April 2013}

\section{Reference}

1. Adamzik M, Görlinger K, Peters J, Hartmann M: Whole blood impedance aggregometry as a biomarker for the diagnosis and prognosis of severe sepsis. Crit Care 2012, 16:R204.

doi:10.1186/cc12583

Cite this article as: Simm V AN, et al:: Whole blood impedance aggregometry findings in experimental endotoxinemia. Critical Care 2013, 17:427.

*Correspondence: matthias.hartmann@uni-due.de

Klinik für Anästhesiologie und Intensivmedizin, Universitätsklinikum Essen, Hufelandstr. 55, D-45122 Essen, Germany 


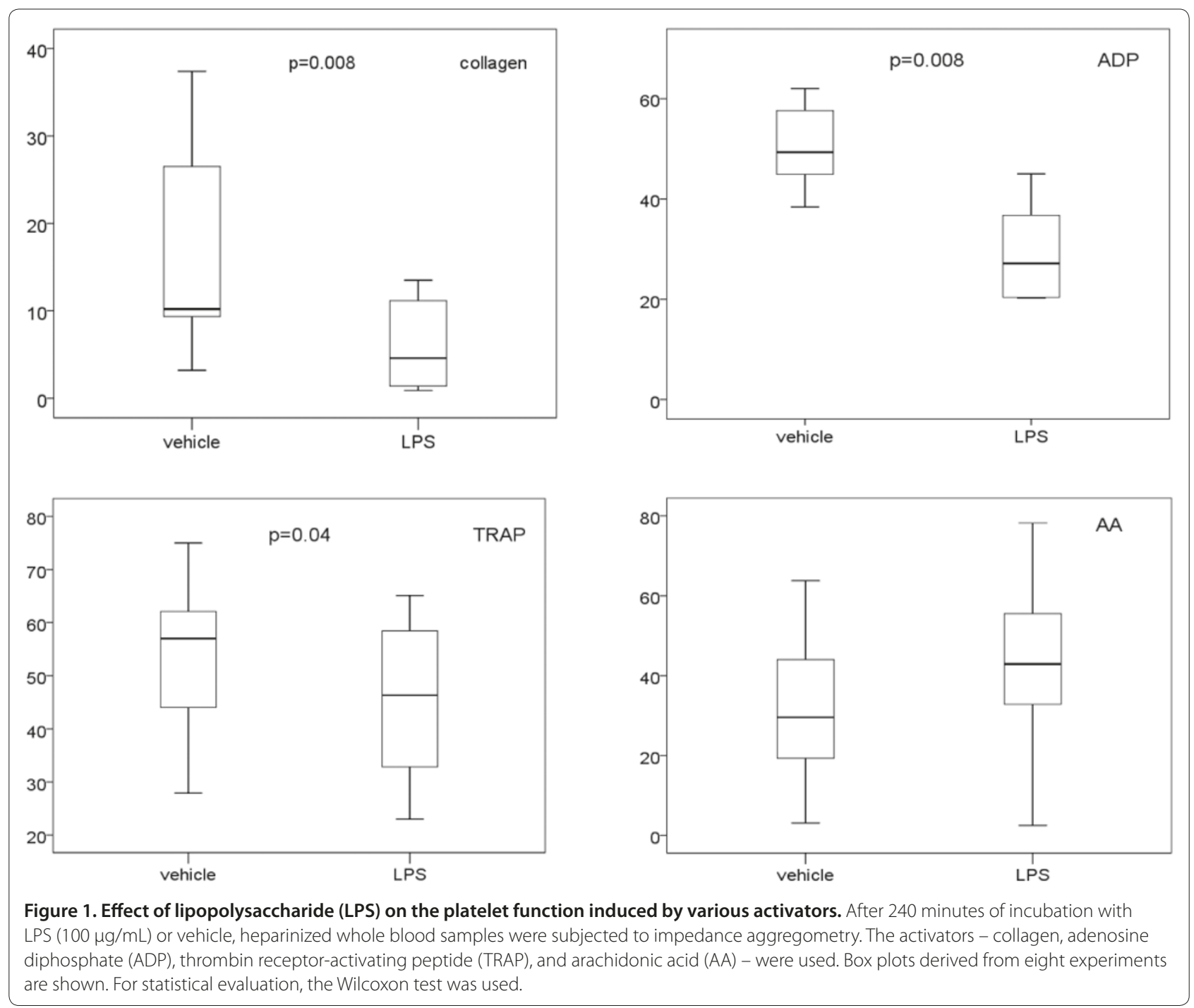


